\title{
IMAGE ANALYSIS OF EGYPTIAN MUMMY HAIR
}

\author{
T. B. Ball,* W. Griggs, ${ }^{*}$ M. Kuchar** R. Phillips*** and W. M. Hess**** \\ *Department of Ancient Scripture, Brigham Young University, Provo, UT, 84602 \\ **Department of Chemistry \& Biochemistry,Brigham Young University, Provo, UT, \\ 84602 \\ ***Department of Geology, Brigham Young University, Provo, UT, 84602 \\ ****Department of Integrative Biology, Brigham Young University, Provo UT, 84602
}

Image analysis can be a means to effectively elucidate morphological differences with both light $[1,2]$ and electron microscopy.[3,4] These procedures have been used to evaluate morphological differences of cross sections of bundles of hair to determine gender differences[3] and morphological variations of caucasoid hair.[4] The purpose of the present investigation was to use image analysis to determine whether morphological differences could be demonstrated between head hair from Egyptian mummies from Fag el Gamous, Fayum, compared with living residents from the same region of Egypt (Mediterranean) as well as caucasoid and oriental hair.

Hair samples were cleaned and processed in resin as previously described [1] which involved sonication in acetone to remove oil, and embedment in Spurr resin.[5] Sections of resin embedded hair were stained in aqueous $1 \%$ toluidine blue $\mathrm{O}, 1 \%$ azure II, and $1 \%$ sodium carbonate. Staining time varied. Mummy hair stained in less than $1 \mathrm{~min}$, but freshly grown hair required up to $5 \mathrm{~min}$. Images were projected using a Leitz Wezlar Prado Universal projector, equipped with a micro attachment. The projected images were recorded and numbered in an orientation so individual hairs were separated so merging of images did not occur.. For each sample, 50 randomly selected individual hair cross-sections were recorded. The images were scanned by an image analyzer. The image analyzer detected 63 shades of gray. The range between 50 and 63 was selected for analysis.

Images were analyzed using ANOVA,Tukey HSD Multiple Comparison tests and Discrimination Analysis to study hair from 9 male and 15 female mummies and two mummies of unknown sex. Hair from 18 caucasoid males and 13 caucasoid females and 4 Chinese were also studied. Morphometric analyses of images were performed using a Macintosh Centris 650 frame grabber board and "Prism" image analysis software. Eighteen morphometric parameters relative to size and shape were evaluated as follows. Area (AREA) is the simple area of the feature. Convex area (CARE) is the area within a taut-string around a feature. Perimeter (PERI) is the length of the feature boundary. Convex perimeter (CPER) is the length of a taut-string around the feature. Length (LENG) is the longest cord within the feature. Breadth (BREA) is the minimum caliper diameter of the feature. Fiber length (FIBE) is the length of the feature along its medial axis. Width (WIDT) is the minor dimension of the feature. Equivalent diam (EQUI) is the diam of a circle with the same area of the feature. Inscribed radius (NSCS) is the radius of the largest circle that can be drawn in the feature. Form factor (FORM) is $4 \pi x$ area/perimeter. This is 1.0 for a perfect circle and diminishes for irregular shapes. 
Roundness (ROUN) is $4 \mathrm{x}$ area/ $\pi \mathrm{x}$ length2. This is also 1.0 for a perfect circle and diminishes with elongation of the feature. Convexity (CONV) is the ratio of convex perimeter to perimeter. It is 1.0 for perfectly convex shape, and diminishes if there are surface indentations. Solidity (SOLI) is the ratio of area to convex area. This is 1.0 for a perfectly convex shape. It diminishes if there are surface indentations. Compactness (COMP) is the equivalent diam/length. Aspect ratio (ASPE) is length/width. Elongation (ELON) is fiber length/width. Curl (CURL) is length/fiber width.

Tests for adequate sample size indicate that twenty five hairs from 25 individuals was an adequate sample size to estimate within $10 \%$ of the population mean at a $95 \%$ confidence level for any of the parameters considered in this study. The results of the Tukey tests showed that parameters of size are significantly different between Mediterranean, caucasoid, and oriental races. Exceptions were LENG and FIBR. Parameters of shape were often significantly different between caucasoid, oriental and Mediterranean races. Exceptions were CONV, SOLI, and CURL. The mummy hair most resembled caucasoid hair in terms of size, but most resembled Mediterranean hair in terms of shape. Female hair and male hair from individuals of the same race were generally significantly different in parameters of size, but not significantly different in parameters of shape.

With discriminant analysis the best model for discriminating between races used all 10 of the parameters of size, and shape parameters of FORM, ROUN, COMP, ASPE, and ELON. Mummy hair is equally similar to Mediterranean and caucasoid hair, but very dissimilar to oriental hair. When mummy hair was considered as a unique race and analyzed as such the results indicated that a majority of the mummy hair could not be correctly discriminated. In studies of sex of the mummy hair $58 \%$ of the males were correctly identified and $69 \%$ of the female hair was correctly discriminated. Studies of sex with Caucasoid hair indicted that $58 \%$ of males nd $68 \%$ of females were correctly discriminated. The overall conclusions are that there are significant morphometric differences between hair taken from different racial types, and from different sexes within the racial types.

\section{References}

[1] W.M. Hess and R.E. Seegmiller, Trans. Am. Microsc. Soc. 107 (1988) 421.

[2] T.B. Ball and W.M. Hess, Microsc Microanal. (1993), 216.

[3] D.A. Johnson et al., Mycologia 91 (1999) 1016.

[4] W.M.Hess et al., Scanning Microsc. 4 (1990) 375.

[5] A.R. Spurr, Ultrastruct. Res. 26 (1969) 31. 
923CD

https://doi.org/10.1017/S143192760210729X Published online by Cambridge University Press 